Ophthalmologe 2022 $\cdot 119: 591-598$ https://doi.org/10.1007/s00347-021-01533-y Eingegangen: 12. August 2021 Überarbeitet: 14. Oktober 2021 Angenommen: 27. Oktober 2021 Online publiziert: 24. November 2021 (c) Der/die Autor(en) 2021

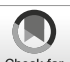

Check for
updates

\section{Phakovitrektomie - Einfluss des Zeitpunktes der Kunstlinsenimplantation auf das korneale Endothel}

\author{
Anastasia Seifert ${ }^{1,2,6}$ (D) $\cdot$ Berthold Seitz ${ }^{3} \cdot$ Gudrun Wagenpfeil ${ }^{4} \cdot$ Klaus Ludwig $^{1,2,5} \cdot$ \\ Matthias Krause ${ }^{1,5}$ \\ ${ }^{1}$ Augenklinik des Klinikums Fürth, Fürth, Deutschland \\ ${ }^{2}$ MVZ für Augenheilkunde und Anästhesie Fürth, Fürth, Deutschland \\ ${ }^{3}$ Klinik für Augenheilkunde, Universitätsklinikum des Saarlandes (UKS), Homburg, Deutschland \\ ${ }^{4}$ Institut für medizinische Biometrie, Epidemiologie und Medizinische Informatik (IMBEI), Universität des \\ Saarlandes, Homburg, Deutschland \\ ${ }^{5}$ MVZ Augenheilkunde Nürnberg, Nürnberg, Deutschland \\ ${ }^{6}$ Universitätsklinikum des Saarlandes und Medizinische Fakultät der Universität des Saarlandes, Homburg, \\ Deutschland
}

Die Autoren Klaus Ludwig und Matthias Krause haben zu gleichen Teilen zu der Arbeit beigetragen.

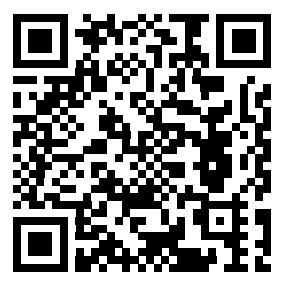

QR-Code scannen \&Beitrag online lesen

\section{Zusammenfassung}

Hintergrund und Ziele: Bei der Phakovitrektomie wird in der Regel zuerst die Katarakt operiert und die Kunstlinse meist vor Beginn der Vitrektomie implantiert. Man kann die Kunstlinse aber auch erst am Ende der Vitrektomie in den Kapselsack implantieren. Damit entfallen optische Beeinträchtigungen durch Hornhauttrübungen und Linsenrand während der Vitrektomie, und Komplikationen wie periphere Netzhautrisse oder Intraokularlinse-Subluxation lassen sich leichter vermeiden. Da diese Variante aber das Hornhautendothel zusätzlich belasten könnte, war das Ziel der vorliegenden Studie, den postoperativen Endothelzellverlust nach Standardphakovitrektomie mit dem nach der alternativen Variante zu vergleichen.

Methoden: In dieser retrospektiven Studie entfielen je 41 Augen auf Gruppe I (Standardphakovitrektomie) oder Gruppe II (Variante). Primäre Zielgrößen waren der absolute und relative Hornhautendothelzellverlust von prä- nach $5 \pm 1$ Wochen postoperativ. Zu den sekundären Zielgrößen gehörten Sehschärfe, Augeninnendruck, Variationskoeffizient der Zellfläche des Endothels (CV), Anteil der hexagonalen Zellform des Endothels (6A), Pachymetrie, intra- und postoperative Komplikationen.

Ergebnisse: Der absolute und der relative Endothelzellverlust in Gruppe I (-108 146 bzw. $-4,1 \pm 5,7 \%)$ unterschieden sich nicht signifikant von dem in Gruppe II $(-73 \pm 122$ bzw. $-3,1 \pm 5,3 \%)(p=0,299 ; p=0,388)$. Auch die sekundären Zielgrößen zeigten keine signifikanten Unterschiede.

Schlussfolgerungen: Die hier vorgestellte Variante der Phakovitrektomie erweitert die chirurgischen Optionen und zeigt im Vergleich zum Standardverfahren keinen signifikant abweichenden postoperativen kornealen Endothelzellverlust.

\section{Schlüsselwörter}

Phakovitrektomie · Endothelzellverlust · Variationskoeffizient der Zellfläche (CV) · Anteil der hexagonalen Zellen $(6 \mathrm{~A} \%) \cdot$ Pachymetrie 


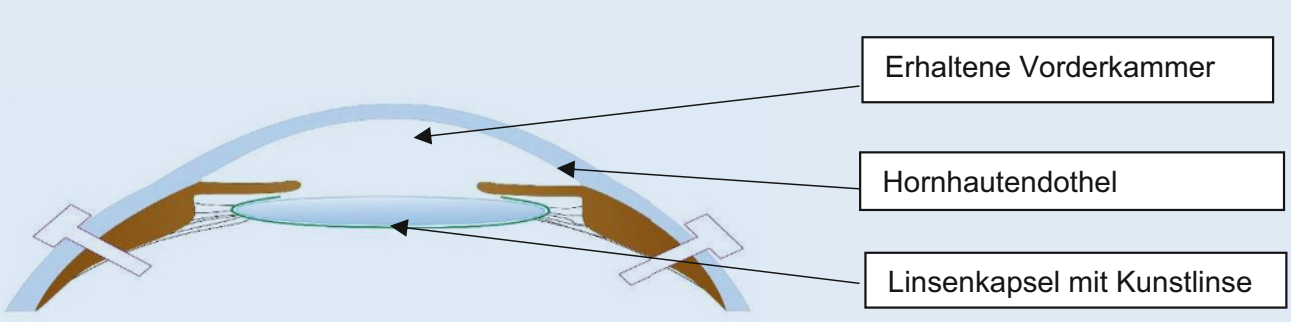

a

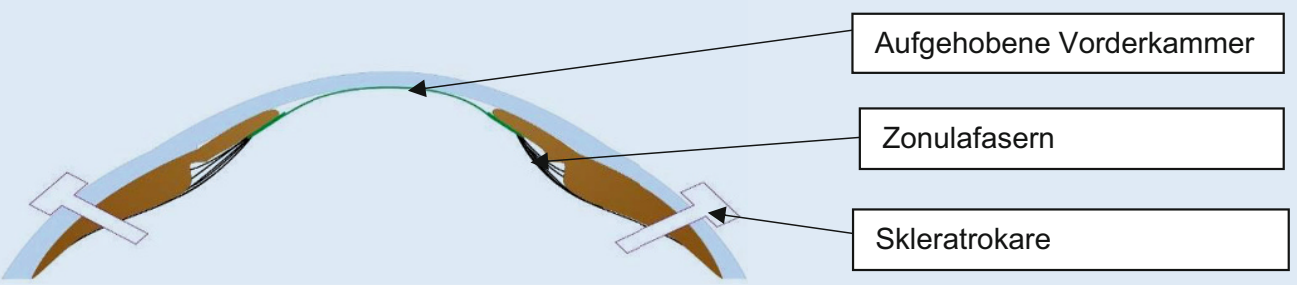

b

Viele Patienten mit Netzhaut-Glaskörper-Erkrankungen leiden auch unter Linsentrübungen, sodass neben dem vitreoretinalen Eingriff eine KataraktOperation (OP) erforderlich ist. Die alternative Variante des kombinierten Vorgehens - Vitrektomie mit am Anfang aufgehobener Vorderkammer und Implantation der Kunstlinse erst zum Ende des vitreoretinalen Eingriffs - bietet dem Operateur Vorteile, könnte aber die Hornhaut belasten. Diese Arbeit soll klären, ob der Endothelkontakt mit der Linsenkapsel zu einem signifikant unterschiedlichen Endothelzellverlust gegenüber dem Standardverfahren führt.

\section{Hintergrund und Fragestellung}

Die Pars-plana-Vitrektomie wird bei phaken Augen gerne mit einer Katarakt-OP kombiniert, denn für die Vitrektomie ist optimale Sicht auf die Netzhaut essenziell $[13,17]$. Auch ist eine vollständige Entfernung des Glaskörpers oft nicht möglich, ohne die Linse dabei zu berühren, was zu ihrer sofortigen Eintrübung führt. Selbst ohne Berührung trübt sich die Linse in über $50 \%$ der Fälle nach einer Pars-plana-Vitrektomie (PPV) innerhalb von 1 bis 2 Jahren ein $[8,14]$. Am häufigsten wird heute der simultane Eingriff durchgeführt [17], indem zunächst die vollständige Lin-
sen-OP unter Ultraschallanwendung samt Implantation einer Kunstlinse erfolgt, und anschließend die vollständige Vitrektomie. Dieses Vorgehen hat aber einige Tücken. So müssen aufgrund der nachfolgenden Vitrektomie alle Kleinschnitte zur LinsenOP gut abgedichtet werden, was zu optisch störenden Hornhauttrübungen bei der Vitrektomie führen kann. Obendrein stört der Rand der Kunstlinse die Sicht auf die äußere Netzhaut. Daher entwickelte einer der Chirurgen unserer Gruppe (K. L.) eine Variante des Standardverfahrens, bei der die Kunstlinse kurz vor Ende der Vitrektomie eingesetzt wird. Durch diese Variante entfallen die genannten optischen Beeinträchtigungen. Der verbesserte periphere Einblick erleichtert zudem die intraoperative Diagnose und Behandlung von Netzhautdefekten. Eine intraoperative Bulbusindentation wird weitgehend überflüssig, sodass eine Scherbelastung der Augenhüllen mit Zonulastress weitgehend vermieden wird. Die vorliegende Arbeit untersucht, ob der Endothelkontakt mit der Linsenkapsel bei der vorgestellten Variante der Phakovitrektomie im Vergleich zum herkömmlichen Verfahren einen zusätzlichen Endothelzellverlust bewirkt (• Abb. 1).
Abb. $1 \varangle$ Schematische Darstellung a der erhaltenen Vorderkammer ohne Endothelkontakt mit der Linsenkapsel bei der Standardphakovitrektomie (Gruppe I), b der aufgehobenen Vorderkammer mit Kontakt zwischen Hornhautendothel und Linsenkapsel bei der Variante der Phakovitrektomie (Gruppe II). Grün: Linsenkapsel, schwarz: Zonulafasern, hellbraun: Iris und Ziliarkörper. (Abbildung nach einer Zeichnung von Klaus Ludwig, grafisch ausgestaltet durch Marc Müller, Klinik für Augenheilkunde, Universitätsklinikum des Saarlandes, Homburg)

\section{Patienten und Methoden}

In dieser retrospektiven Beobachtungsstudie wurden 82 phake Augen von 79 Patienten eingeschlossen, die zwischen April 2019 und April 2020 mittels standardisierter (Gruppe $1 ; n=41$ ) oder alternativer (Gruppe II; $n=41$ ) Phakovitrektomie (23 und $25 \mathrm{G}$ ) von demselben Operateur (A. S.) operiert worden waren. Eine Vorselektion der Varianten, etwa nach chirurgischen Indikationen oder Komplexität des Eingriffs, war nicht gegeben. In jeder Gruppe entfielen 30 OP-Indikationen (73\%) auf die Diagnosen epiretinale Gliose und 11 (27\%) auf die Diagnosen Makulaforamen, Ablatio retinae, proliferative Vitreoretinopathie oder Glaskörperblutungen. Ausgeschlossen waren Augen mit Pathologien der Zonulafasern, der Hornhaut sowie Augen mit einer Endothelzahl unter 1400 Zellen $/ \mathrm{mm}^{2}$ und einer Bulbusachsenlänge $<19,5 \mathrm{~mm}$ oder $>31,0 \mathrm{~mm}$.

Zunächst wurden drei 23- bzw. 25-GKanülen unter Verschieben der Bindehaut schräg in der Sklera fixiert. Dann erhielt Gruppe I im ersten Teilschritt eine vollständige Kataraktoperation einschließlich Implantation einer Intraokularlinse (IOL) und Versiegelung der Vorderkammerzugänge mittels BSS (Balanced Salt Solution). Anschließend erfolgte im zweiten Teilschritt des kombinierten Eingriffs eine 
vollständige PPV. Bei Gruppe II erfolgte die Katarakt-OP in identischer Weise wie bei Gruppe I bis unmittelbar vor der IOLImplantation. Nun folgte die PPV im aphaken Zustand (bei bewusst aufgehobener Vorderkammer und angestrebtem Kontakt von Hornhautendothel und Linsenkapsel während der vorderen Vitrektomie zu Beginn der PPV); eine Versiegelung mittels BSS war deshalb zunächst nicht erforderlich. Die IOL-Implantation unter Viskoelastikum fand am Ende der PPV statt; erst nach Ausspülen des Viskoelastikums wurden die Vorderkammerzugänge versiegelt.

Bei allen Patienten wurden routinemäßig die korneale Endothelzelldichte, Abweichung von Größe und Form der Endothelzellen und Hornhautdicke im Hornhautzentrum mit dem Endothelzellmessgerät EM-3000 bzw. 4000 (Tomey, Nagoya, Japan) präoperativ sowie $5 \pm 1$ Woche postoperativ gemessen und verglichen. Für die statistischen Auswertungen wurde das Statistik-Programm SPSS Statistics 27,0 (IBM, New York, USA) eingesetzt. Die Daten wurden mittels Mann-WhitneyU-Test, t-Test, exaktem Test von Fischer bzw. Chi-Quadrat-Test oder Wilcoxon-Test ausgewertet, um die Signifikanz der Übereinstimmung beider Gruppen zu überprüfen. Ein $p$-Wert $<0,05$ wurde als statistisch signifikant angesehen.

\section{Ergebnisse}

Die beiden Gruppen zeigten eine gute Vergleichbarkeit ohne statistisch signifikanten Unterschied in der Verteilung von Alter, Geschlecht, Diagnose sowie Achsenlänge und Vorderkammertiefe (• Tab. 1).

Bei keinem gemessenen OP-Parameter ließ sich eine statistisch signifikante Differenz zwischen den Gruppen I und II nachweisen (-Tab. 2). Die Mittelwerte aller Parameter waren ebenso wie die Standardabweichungen in beiden Gruppen ähnlich; lediglich die Phakoleistung war in Gruppe II $(14,4 \pm 2,8 \%)$ im Mittel tendenziell etwas höher als in Gruppe I (13,8 $23,7 \%$; $p=0,082$ ).

Die Sehschärfe (MW \pm STD) betrug präoperativ in Gruppe I 0,37 $\pm 0,2$, in Gruppe II 0,36 $\pm 0,2(p=0,640)$, postoperativ in Gruppe I 0,61 $\pm 0,2$ und in Gruppe II $0,58 \pm 0,3(p=0,884)$ ohne statistisch signi- fikanten Unterschied zwischen den beiden Gruppen $(p=1,000)$.

Der Mittelwert des Augeninnendruckes lag nach der OP um $0,8 \pm 4,2 \mathrm{~mm} \mathrm{Hg}$ in Gruppe I und um 1,7 $\pm 3,8 \mathrm{~mm} \mathrm{Hg}$ in Gruppe II niedriger als vor der OP ohne statistisch signifikanten Unterschied, auch bezüglich der absoluten bzw. relativen Änderung des Augeninnendruckes ( $p=0,394$, $p=0,361$ ) (• Tab. 3; • Abb. 2).

Der Mittelwert der präoperativen Endothelzelldichte betrug in Gruppe I $2520 \pm 304$ Zellen $/ \mathrm{mm}^{2}$ und nahm signifikant auf $2412 \pm 289$ Zellen $/ \mathrm{mm}^{2}$ ab $(p<0,001)$, entsprechend einem relativen Verlust von $-4,1 \pm 5,7 \%$. In Gruppe II sank die präoperative Endothelzelldichte von $2415 \pm 246$ Zellen $/ \mathrm{mm}^{2}$ auf $2343 \pm 277$ Zellen $/ \mathrm{mm}^{2}$ (• Abb. 3) $(p=0,001)$ mit einem relativen Verlust von $-3,1 \pm 5,4 \%$. Beim Vergleich der Gruppen ergab sich kein statistisch signifikanter Unterschied der Endothelzelldichte bei präoperativen $(p=0,081)$ wie postoperativen $(p=0,239)$ Werten. Die Endothelzelldichte nahm absolut in Gruppe I um $-108 \pm 146$ Zellen $/ \mathrm{mm}^{2}$ und Gruppe II um $-73 \pm 123$ Zellen $/ \mathrm{mm}^{2}$ ab $(p=0,299)$

\section{Hier steht eine Anzeige.}


Tab. 1 Verteilung von Alter, Geschlecht, Augen, Achsenlänge, Vorderkammertiefe und Diagnosen zwischen den Gruppen I und II

\begin{tabular}{|l|l|l|l|}
\hline Gruppe & I $(n=41)$ & II $(n=41)$ & $p$-Wert \\
\hline Alter (MW \pm STD) & $68 \pm 7,5$ & $70 \pm 8$ & $p^{1}=0,258$ \\
\hline Geschlecht (weiblich/männlich) & $17 / 24$ & $18 / 23$ & $p^{2}=1,000$ \\
\hline Augen (rechts/links) & $17 / 24$ & $15 / 26$ & $p^{2}=0,821$ \\
\hline Achsenlänge (mm) (MW \pm STD) & $24,0 \pm 1,2$ & $23,5 \pm 1,2$ & $p^{3}=0,068$ \\
\hline Vorderkammertiefe (mm) (MW \pm STD) & $3,1 \pm 0,4$ & $3,1 \pm 0,4$ & $p^{3}=0,930$ \\
\hline Diagnose (Gliose/Makulaforamen/Sonstiges) & $30 / 7 / 4$ & $30 / 4 / 7$ & $p^{2}=0,453$ \\
\hline
\end{tabular}

$p^{1}$ t-Test; $p^{2}$ exakter Test nach Fischer; $p^{3}$ Mann-Whitney-U-Test

Sonstiges: Ablatio retinae, proliferative Vitreoretinopathie und Glaskörperblutungen

$M W \pm S T D$ Mittelwert und Standardabweichung

Tab. 2 Mittelwert der effektiven Phakozeit, Phakoleistung, gesamten Phakozeit, Katarakt- und Vitrektomiezeit in den Gruppen I und II

\begin{tabular}{|l|l|l|l|}
\hline Gruppe & I $(n=41)$ & II $(n=41)$ & $p$-Wert \\
\hline Effektive Phakozeit (s) & $5,1 \pm 2,4$ & $4,9 \pm 1,8$ & 0,842 \\
\hline Phakoleistung (\%) & $13,8 \pm 3,7$ & $14,4 \pm 2,8$ & 0,082 \\
\hline Gesamte Phakozeit (s) & $35,7 \pm 13,5$ & $32,6 \pm 11$ & 0,349 \\
\hline Katarakt-OP-Zeit ohne IOL (min) & $6,3 \pm 1,3$ & $6,4 \pm 1,1$ & 0,498 \\
\hline PPV-Zeit (min) & $32,5 \pm 17$ & $32,3 \pm 10$ & 0,516 \\
\hline $\begin{array}{l}\text { PPV-Zeit schließt IOL-Implantation ein } \\
\text { PPV Pars-plana-Vitrektomie, IOL Intraokularlinse } \\
\text { a Mann-Whitney-U-Test für unverbundene Stichproben }\end{array}$ & \\
\hline
\end{tabular}

\begin{tabular}{|c|c|c|c|c|c|}
\hline Gruppe & $(n=41)$ & Präoperativ & Postoperativ & $\begin{array}{l}\text { Absolute } \\
\text { Änderung }\end{array}$ & $\begin{array}{l}\text { Relative } \\
\text { Änderung }\end{array}$ \\
\hline Endothelzellzahl & 1 & $2520 \pm 304$ & $2412 \pm 289$ & $-108 \pm 146$ & $-4,1 \pm 5,7$ \\
\hline (Zellen/mm²) & II & $2415 \pm 246$ & $2343 \pm 277$ & $-73 \pm 123$ & $-3,1 \pm 5,4$ \\
\hline- & $p$-Wert ${ }^{d}$ & 0,081 & 0,239 & 0,299 & 0,388 \\
\hline$C V^{a}(\%)$ & 1 & $40,6 \pm 8,0$ & $41,5 \pm 7,8$ & $-0,93 \pm 9,3$ & $-4,7 \pm 22,7$ \\
\hline- & II & $38,3 \pm 7,8$ & $41,3 \pm 9,8$ & $-2,92 \pm 9,9$ & $-9,6 \pm 24,7$ \\
\hline- & $p$-Wert ${ }^{d}$ & 0,126 & 0,603 & 0,200 & 0,190 \\
\hline $6 A^{b}(\%)$ & I & $44,1 \pm 9,1$ & $43,6 \pm 6,9$ & $-0,4 \pm 8,2$ & $-1,8 \pm 20,0$ \\
\hline- & II & $47,9 \pm 7,2$ & $44,2 \pm 7,0$ & $-3,7 \pm 8,3$ & $6,1 \pm 18,0$ \\
\hline- & $p$-Wert ${ }^{d}$ & 0,015 & 0,723 & 0,097 & 0,088 \\
\hline Pachymetrie & I & $544 \pm 35$ & $547 \pm 39$ & $+3,3 \pm 16$ & $+0,6 \pm 3$ \\
\hline$(\mu \mathrm{m})$ & II & $542 \pm 30$ & $545 \pm 38$ & $+2,6 \pm 17$ & $+0,4 \pm 3$ \\
\hline- & $p$-Wert ${ }^{d}$ & 0,670 & 0,393 & 0,948 & 0,919 \\
\hline $\mathrm{IOD}^{\mathrm{C}}(\mathrm{mm} \mathrm{Hg})$ & I & $15,7 \pm 3$ & $14,9 \pm 4$ & $-0,8 \pm 4,2$ & $-2,7 \pm 26$ \\
\hline- & II & $15,2 \pm 4$ & $13,5 \pm 3$ & $-1,7 \pm 3,8$ & $-8,4 \pm 22$ \\
\hline- & $p$-Wert ${ }^{d}$ & 0,331 & 0,099 & 0,394 & 0,361 \\
\hline \multicolumn{6}{|c|}{$\begin{array}{l}{ }^{a} \text { CV: Variationskoeffizient der Zellfläche } \\
{ }^{b} 6 \text { A: Anteil hexagonaler Zellen } \\
{ }^{c} \text { IOD: Augeninnendruck } \\
{ }^{d} \text { Mann-Whitney-U-Test für unverbundenen Stichproben }\end{array}$} \\
\hline
\end{tabular}

(• Tab. 3). Der Änderung des Variationskoeffizienten der Zellfläche (CV) $(p=0,200)$, der Anzahl der hexagonalen Zellen (6A) $(p=0,097)$ und des Mittelwertes der Pachymetrie $(p=0,948)$ war zwischen den Gruppen ohne statistisch signifikanten Unterschied (• Tab. 3). Intraoperativ fanden sich in der Gruppe I bei einem Patienten $(2,4 \%)$ eine Amotio retinae und bei einem weiteren Patienten (2,4\%) beim Eindellen ein Irisprolaps durch den Phakotunnel, sodass eine Kreuzstichnaht mit Nylon 10,00 erforderlich war ( $p=0,724)$ (• Abb. 4). Die Häufigkeit postoperativer Komplikationen unterschied sich in beiden Gruppen nicht $(p=0,958)$.

\section{Diskussion}

Für die Transparenz der Hornhaut ist das Hornhautendothel entscheidend. Physiologisch nimmt die Endothelzelldichte in der Normalbevölkerung um ca. 0,6\% pro Jahr ab [1]. Eine Endothelzelldekompensation ist bei zentralen Endotheldichten von 700 Zellen $/ \mathrm{mm}^{2}$ und darunter zu erwarten [4]. Durch jede Phakoemulsifikation der Linse und auch durch Phakovitrektomie wird es traumatisiert. Folglich will man chirurgisches Endothelzelltrauma möglichst minimieren. Die vorliegende Studie vergleicht die Veränderung des zentralen Hornhautendothels prä- vs. postoperativ zwischen der klassischen Technik der Phakovitrektomie und einer alternativen Variante mit IOL-Implantation am Ende der Vitrektomie. Trotz des zusätzlichen Endothelkontaktes der Linsenkapsel in Gruppe II zeigte diese weder einen signifikant anderen Endothelzellverlust noch andere klinische Zeichen eines zusätzlichen Hornhauttraumas im Vergleich zu Gruppe I. Die postoperative Endothelzellabnahme nach Katarakt-OP wird in der Literatur zwischen 4 und $25 \%$ beschrieben [2, 3, $11,12,18]$. Frieberg et al. berichteten über einen Endothelzellverlust nach einfacher Vitrektomie von $1,3 \pm 1,4 \%$, bei Vitrektomie in aphaken Augen von 12,6 2,3\% und bei aphaken Augen nach Flüssigkeits-

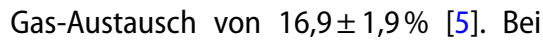
Vitrektomie ohne Katarakt-OP, jedoch mit Silikonölendotamponade erreichte der Endothelzellverlust $5 \%$ innerhalb 12 Monaten [7]. Die angegebenen Zahlen lassen sich dahingehend deuten, dass die Exis- 

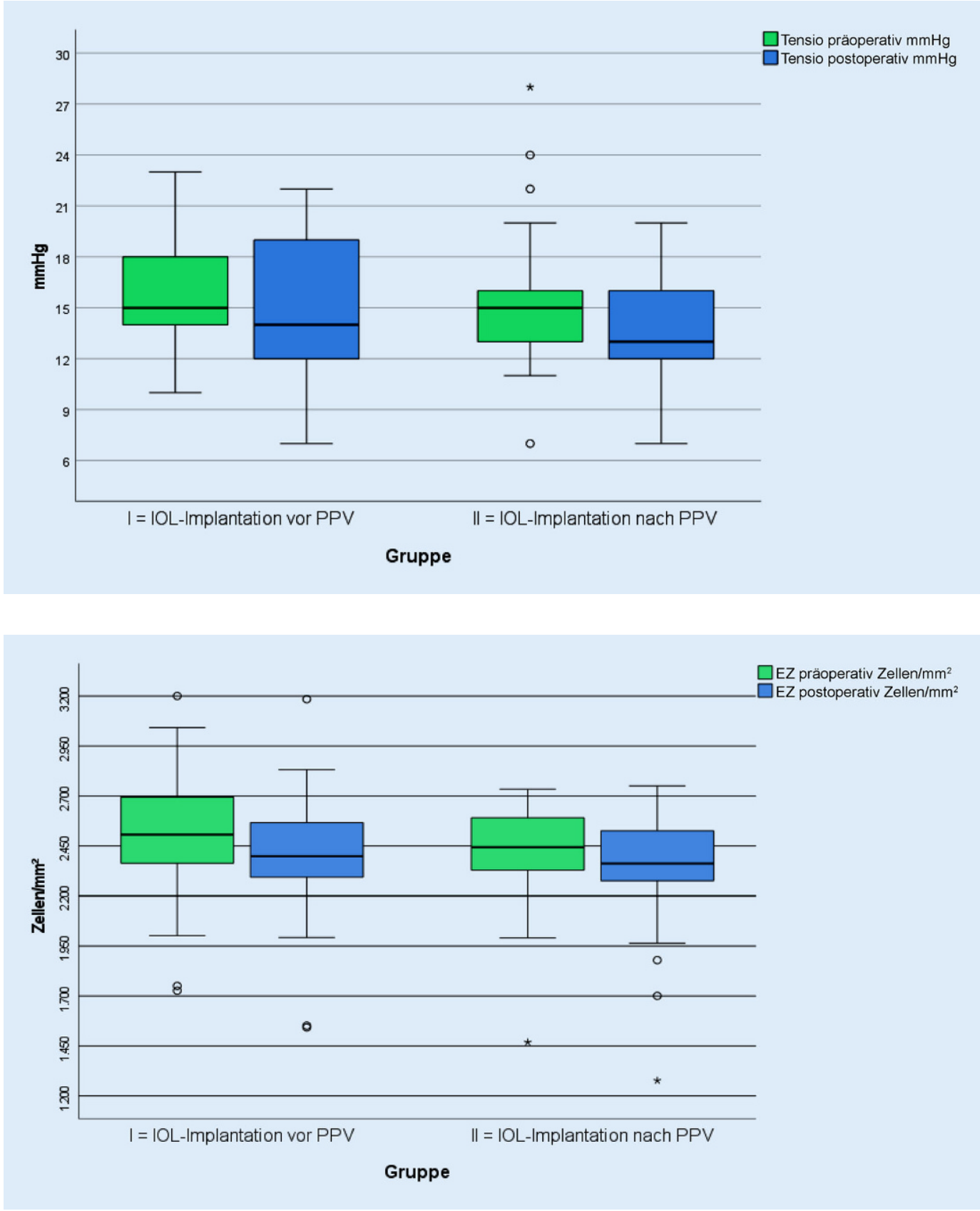

Abb. $2 \varangle$ Vergleich des präoperativen und postoperativen Augeninnendruckes (Tensio) in den Gruppen I und II. PPV Parsplana-Vitrektomie, IOL Intraokularlinse, Kreise Ausreißer, Sterne Extremwerte
Abb. $3 \triangleleft$ Vergleich der präoperativen und postoperativen Endothelzelldichte (EZ) zwischen den Gruppen I und II. PPVPars-planaVitrektomie, IOL Intraokularlinse, EZ Endothelzelldichte. Kreise Ausreißer, Sterne Extremwerte tenz einer eigenen oder künstlichen Linse während der Vitrektomie die Endothelzellen effektiv schützt, besonders dann, wenn eine Endotamponade notwendig wurde. Die Ergebnisse unserer Arbeit sprechen dafür, dass eine solche Protektion der Endothelzellen auch allein durch die intakte Linsenkapsel ohne implantierte Linse trotz des initialen Endothel-KapselKontakts gegeben ist. Anders ausgedrückt unterschied sich der Endothelzellverlust während einer PPV mit Kontakt von Linsenkapsel und Hornhautendothel nicht signifikant von dem des Standardverfahrens. Zum gegenwärtigen Zeitpunkt existieren in der Literatur keine Daten über den Endothelzellverlust nach Vitrektomie mit IOL-Implantation am OP-Ende.

In der Studie von Li et al. haben die Operateure erst nach Abschluss der OP des hinteren Segments eine IOL implantiert, um eine bessere Visualisation bei der Vitrektomie zu erreichen. Es wurde berichtet, dass sich ein Hornhautödem intraoperativ bei 3,2\% und postoperativ bei $8,1 \%$ aller Fälle entwickelte [13]. In der Studie von Hamoudi et al., die die Endothelzellabnahme nach 12 Monaten zwischen 3 Gruppen (Katarakt-OP $\rightarrow$ Vitrektomie: $15,3 \%$, Vitrektomie $\rightarrow$ KataraktOP: $20 \%$ und Katarakt-OP mit direkt folgender Vitrektomie: 19,3\%) untersuchte, wurden keine signifikanten Unterschiede des Endothelzellverlustes festgestellt [9]. Auch diese Ergebnisse sprechen dafür, dass die Endothelzellabnahme nach dem derzeitigen Stand der Literatur nicht mit dem ausgewählten operativen Verfahren korreliert und somit die Entscheidung 


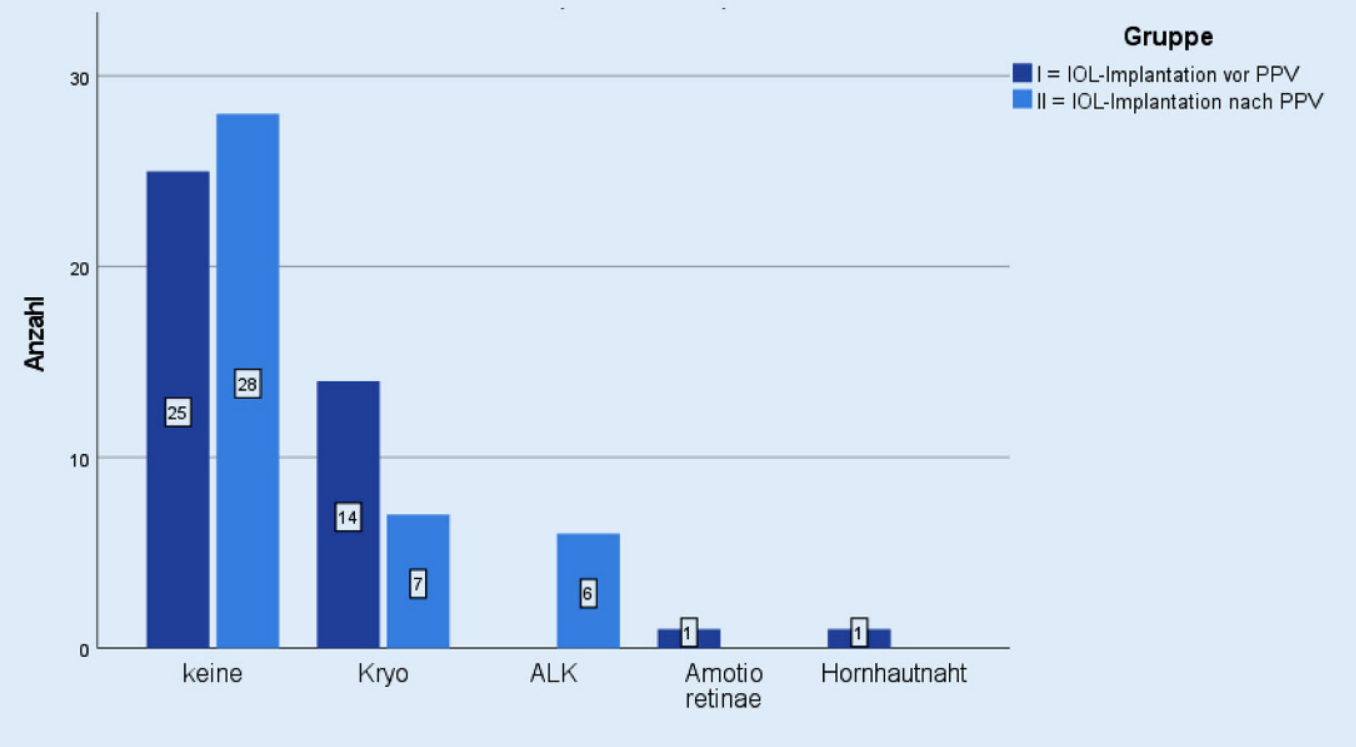

Abb. $4 \varangle$ Vergleich der intraoperativen Komplikationen in den Gruppen I und II. ALK Argonlaserkoagulation, Kryo Kryoretinopexie, PPVPars-plana-Vitrektomie, IOL Intraokularlinse für oder gegen eines der geplanten OPVerfahren nicht in erster Linie vom Grad des Endothelzellverlusts abhängt.

Auch bei den sekundären Endpunkten der vorgelegten Studie zeigte die Vitrektomie ohne vorimplantierte IOL keinerlei Nachteil. Die erhebliche Verbesserung der mittleren korrigierten Sehschärfe in beiden Gruppen ist sicherlich überwiegend auf die Kataraktoperation im Rahmen des Gesamteingriffs zurückzuführen. Der postoperative Mittelwert nach $5 \pm 1$ Woche von ca. 0,6 in beiden Gruppen erklärt sich zudem aus den visusbegrenzenden präoperativen Diagnosen und der bei der postoperativen Kontrolle noch nicht abgeschlossenen Visuserholung. Zwischen beiden Gruppen zeigte sich kein signifikanter Unterschied. Die Veränderung des Augeninnendrucks in Gruppe I und II (• Tab. 3) weist trotz fehlender Signifikanz 2 interessante Phänomene auf. Zum einen war die mittlere Drucksenkung in Gruppe II mit $1,7 \mathrm{~mm} \mathrm{Hg}$ höher als die in Gruppe I mit $0,8 \mathrm{mmHg}$. Der zugehörige Boxplot (- Abb. 2) zeigt auch, dass die Druckwerte in Gruppe II postoperativ geringer streuten als in Gruppe I. Ob dies auf eine evtl. Schutzwirkung des Trabekelwerks durch die zeitweise aufgehobene Vorderkammer in Gruppe II hinweisen könnte, wäre nur in einer wesentlich umfangreicheren prospektiven Studie zu klären. In der vorliegenden Studie zeigte sich bei keinem der untersuchten Parameter eine signifikante
Differenz zwischen den Gruppen präoperativ sowie postoperativ.

Beide Kollektive mit je 41 Augen waren sehr gut vergleichbar. Weder hinsichtlich ihrer demografischen Struktur noch der präoperativen OP-Indikationen, noch der ophthalmologischen Messwerte, noch der intraoperativen Parameter gab es kritische Unterschiede. Die Vitrektomie im Aphakiezustand wies vergleichbare Ergebnisse bei ähnlichen Komplikationsraten ohne statistisch signifikante Differenz auf. In Gruppe I erhielten aufgrund peripherer retinaler $\mathrm{Pa}$ thologien 14 von 41 Patienten (34,1\%) und in Gruppe II 13 von 41 Patienten (31,7\%) eine Retinopexie. Die Literaturrecherche zeigte ähnliche Häufigkeiten für die Kryopexie (30\%) und sogar einen höheren Anteil für die Laserretinopexie $51 \%[6,19]$. Interessant ist, dass bei Gruppe I in allen 14 Fällen eine Kryoretinopexie nötig war, dagegen nur in 7 der 13 Fälle aus Gruppe II. In den restlichen 6 Fällen erlaubte hier der bessere periphere Funduseinblick ohne optische Störung eine Laserretinopexie ohne Eindellung. Die Entscheidung zugunsten einer Kryo- und gegen die Laserretinopexie in der Gruppe der Patienten mit initialer IOL-Implantation spricht nach Ansicht der Autoren dafür, dass in dieser Gruppe I verfahrensbedingt meist nur nach Eindellung des äußeren Teils der Netzhaut alle behandlungsbedürftigen Areale sichtbar waren. Die Laserkoagulation gilt im Vergleich zur Kryokoagulation als schonender, weil Letztere einen zeitlich und räum- lich umschriebenen Zusammenbruch der Blut-Retina-Schranke verursacht, der wiederum eine erhöhte Entzündungsreaktion nach sich ziehen kann [10, 15, 16]. Zudem stellt die Kryoretinopexie mit Eindellung eine Belastung für die Zonulaarchitektur dar. Die Phakovitrektomievariante in Gruppe II ermöglichte also wahrscheinlich gegenüber dem bisherigen Standardverfahren eine schonendere Sanierung peripherer retinaler Pathologien.

Eine Reizmiosis beim Kollabieren der Vorderkammer wurde nicht als auffällig und störend wahrgenommen. Die IOL-Implantation konnte in allen Fällen regelrecht erfolgen. Bei keinem der Patienten gab es intra- oder postoperativ ( $5 \pm 1$ Wochen) einen Hinweis auf eine IOL-Dislokation.

\section{Limitationen}

In dieser Studie gab es einige Einschränkungen. Die Endothelzellmessung wurde nur in der zentralen Hornhaut vorgenommen. Auch die Pupillenweite, der Grad der Katarakt und die Zeit bis zur spontanen Wiederherstellung der Vorderkammer wurden nicht erfasst. Die Studie erfolgte retrospektiv und mit einer begrenzten $\mathrm{Pa}$ tientenzahl.

\section{Fazit für die Praxis}

- Eine alternative Variante der Phakovitrektomie wird vorgestellt (Vitrektomieren im aphaken Zustand bei bewusst vorübergehend aufgehobener Vorderkam- 
mer und mit IOL-Implantation am Ende der OP). Methodische Vorteile: optimaler Funduseinblick ohne Abdichten von $\mathrm{Pa}$ razentesen mit konsekutiven störenden Hornhauttrübungen vor Durchführung der PPV; größere Sicherheit im Vermeiden von peripheren Netzhautrissen und ggf. erleichterte Behandlung von peripheren Netzhautrissen bei Verzicht auf Eindellung des Bulbus mit geringerem Zonulastress.

- Kein signifikanter Unterschied bezüglich des Endothelzellverlusts und der anderen gemessenen Parameter im Vergleich zur Standardmethode (IOL-Implantation am Ende der Katarakt-OP vor Beginn der Vitrektomie).

\section{Korrespondenzadresse}

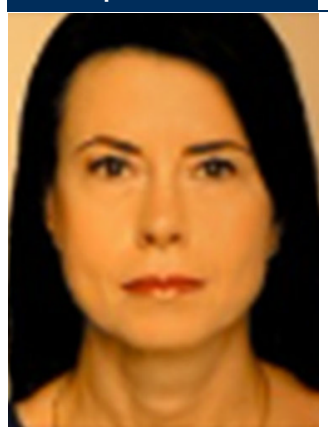

\section{Anastasia Seifert}

Universitätsklinikum des Saarlandes und Medizinische Fakultät der Universität des Saarlandes

Homburg, Deutschland

a.seifert@osg.de

Funding. Open Access funding enabled and organized by Projekt DEAL.

\section{Einhaltung ethischer Richtlinien}

Interessenkonflikt. A. Seifert, B. Seitz, G. Wagenpfeil, K. Ludwig und M. Krause geben an, dass kein Interessenkonflikt besteht.

Für diesen Beitrag wurden von den Autoren keine Studien an Menschen oder Tieren durchgeführt. Für die aufgeführten Studien gelten die jeweils dort angegebenen ethischen Richtlinien.

Open Access. Dieser Artikel wird unter der Creative Commons Namensnennung 4.0 International Lizenz veröffentlicht, welche die Nutzung, Vervielfältigung, Bearbeitung, Verbreitung und Wiedergabe in jeglichem Medium und Format erlaubt, sofern Sie den/die ursprünglichen Autor(en) und die Quelle ordnungsgemäß nennen, einen Link zur Creative Commons Lizenz beifügen und angeben, ob Änderungen vorgenommen wurden.

\section{Phacovitrectomy-Influence of the timing of intraocular lens implantation on the corneal endothelium}

Background and purpose: In phacovitrectomy the cataract is usually operated on first including implantation of the intraocular lens (IOL) before beginning vitrectomy but the IOL can also be implanted following vitrectomy. This variation avoids optical impairments from corneal opacities and the lens rim, improves the visualization of the retina during surgery and might thereby reduce intraoperative complications, such as peripheral retinal tears or IOL subluxation. It might, however, increase stress on the corneal endothelium. The aim of this study was, therefore, to compare postoperative corneal endothelial cell loss for the standard procedure of phacovitrectomy and the surgical variation.

Methods: In this retrospective study 41 eyes were each assigned to group I (standard phacovitrectomy) or group II (variation of phacovitrectomy). The primary endpoint was the absolute and relative corneal endothelial cell loss appearing $5 \pm 1$ weeks postoperatively with reference to the preoperative number of endothelial cells. Secondary endpoints included visual acuity, intraocular pressure, coefficient of variation of endothelial cell area (CV), proportion of hexagonal endothelial cell forms (6A), pachymetry, intraoperative and postoperative complications.

Results: The absolute and relative endothelial cell loss in group I $(-108 \pm 146$;

$-4.1 \pm 5.7 \%)$ did not differ significantly from that in group II $(-73 \pm 122 ;-3.1 \pm 5.3 \%$, $p=0.299 ; p=0.388$ ). The secondary endpoints also showed no significant differences. Conclusion: The presented variation of phacovitrectomy expands the surgical options and does not show a significantly different postoperative corneal endothelial cell loss compared to the standard procedure.

\section{Keywords}

Phacovitrectomy - Endothelial cell loss - Coefficient of variation of cell area (CV) - Proportion of hexagonal cells $(6 \mathrm{~A} \%) \cdot$ Pachymetry

Creative Commons Lizenz, sofern sich aus der Abbildungslegende nichts anderes ergibt. Sofern das betreffende Material nicht unter der genannten Creative Commons Lizenz steht und die betreffende Handlung nicht nach gesetzlichen Vorschriften erlaubt ist, ist für die oben aufgeführten Weiterverwendungen des Materials die Einwilligung des jeweiligen Rechteinhabers einzuholen.

Weitere Details zur Lizenz entnehmen Sie bitte der Lizenzinformation auf http://creativecommons.org/ licenses/by/4.0/deed.de.

\section{Literatur}

1. Bourne WM (2003) Biology of the corneal endothelium in health and disease. Eye 17:912. https://doi.org/10.1038/sj.eye.6700559

2. Cho YK, Chang HS, Kim MS (2010) Risk factors for endothelial cell loss after phacoemulsification: comparison in different anterior chamber depth groups. Korean JOphthalmol 24(1):10-15

3. Cho YK, Chang HS, La TY, Ji D, Kim H, Choi JA, Kim MS (2010) Anterior segment parameters using Pentacam and prediction of corneal endothelial cell loss after cataract surgery. Korean JOphthalmol 24(5):284-290

4. EdelhauserHF(2006) The balance between corneal transparencyand edema the proctor lecture. Invest Ophthalmol Vis Sci 47(5):1755-1767

5. Friberg TR, Doran DL, Lazenby FL (1984) The effect of vitreous and retinal surgery on cor- neal endothelial cell density. Ophthalmology 91(10):1166-1169. https://doi.org/10.1016/ s0161-6420(84)34166-5

6. Garweg JG, Ouassi D, Pfister IB (2020) Hybrid 23/27 Gauge Vitrektomie - Kombination des Charmes von $27 \mathrm{G}$ mit der Wirksamkeit von 23G. Clin Ophthalmol 14:299-305. https://doi.org/10. 2147/OPTH.S233884

7. Goezinn F, Nuijts RM, Liem AT, Lundqvist IJ, Berendschot TJ, Cals DW, La Heij EC (2014) Corneal endothelial cell density after vitrectomy with silicone oil for complex retinal detachments. Retina 34(2):228-236

8. Grajewski L, Grajewski O, Carstens J, Krause L (2021) 25-Gauge-Makulachirurgie im Vergleich mit und ohne kombinierte Phakoemulsifikation und Kunstlinsenimplantation. Ophthalmologe. https://doi.org/10.1007/s00347-021-01461-x

9. Hamoudi H, Christensen UC, La Cour M (2017) Corneal endothelial cell loss and corneal biomechanical characteristics after two-step sequential or combined phaco-vitrectomy surgery for idiopathic epiretinal membrane. Acta Ophthalmol 95(5):493-497

10. Hesse L, Kroll P (1999) Enzymatisch induzierte hintere Glaskörperabhebung bei proliferativer diabetischer Vitreoretinopathie. Klin Monatsbl Augenheilkd 214(02):84-89

11. Ho JW, Afshari NA (2015) Advances in cataract surgery: preserving the corneal endothelium. Curr Opin Ophthalmol 26:22-27

12. Kohlhaas M, Stahlhut O, Tholuck J, Richard G (1997) Entwicklung der Hornhautdicke und Endothelzelldichte nach Kataraktextraktion mittels Pha- 
koemulsifikation. Ophthalmologe 94(7):515-518. https://doi.org/10.1007/s003470050150

13. Li W, Sun G, Wu R, Wang $X$, Xu M, Sun $C$ (2009) Longterm results after phacovitrectomy and foldable intraocular lens implantation. Acta Ophthalmol 87(8):896-900

14. Petermeier K, Szurman P, Bartz-Schmidt U, Gekeler F (2010) Pathophysiologie der KataraktEntwicklung nach Vitrektomie. Klin Monatsbl Augenheilkd 227(03):175-180. https://doi.org/10. 1055/s-0029-1245271

15. Röber H, Göring W, Sous H, Reim M (1977) Concentration of ampicillin in the vitreous after cryocoagulation. Graefes Arch Clin Exp Ophthalmol 204(4):275-280

16. Priglinger S, Siedlecki J, Hattenbach L-O, Grisanti S (2021) Proliferative Vitreoretinopathie (PVR)-Chirurgie:, Scar Wars". Ophthalmologe 118(1):18-23

17. Savastano A, Savastano MC, Barca F, Petrarchini F, Mariotti C, Rizzo S (2014) Combining cataract surgery with 25-gauge high-speed pars plana vitrectomy: results from a retrospective study. Ophthalmology 121:299-304

18. Walkow T, Anders N, Klebe S (2000) Endothelial cell loss after phacoemulsification: relation to preoperative and intraoperative parameters. J Cataract Refract Surg 26(5):727-732. https://doi. org/10.1016/S0886-3350(99)00462-9

19. Wirbelauer C, Häberle H, Pham D (2011) Klinische Erfahrungen zur Färbung der Netzhautoberfläche mit Brilliant blau G. Klin Monatsbl Augenheilkd 228:62-65
Andrea Petermann-Meyer, Jens Panse, Tim H. Brümmendorf

\section{Leben mit Krebs}

Praktischer Ratgeber für Betroffene, Angehörige und Behandelnde

Berlin, Heidelberg: Springer-Verlag GmbH 2021, 1. Auflage, 256 S., 18 Abb., (ISBN: 978-3-662-59165-9), Softcover 20,36 EUR

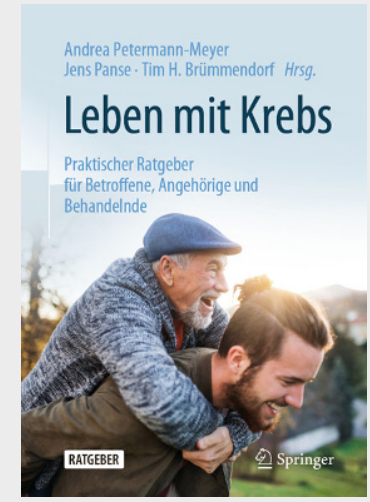

Der Ratgeber „Leben mit Krebs“ richtet sich an Menschen mit Krebserkrankungen und ist das Resultat einer seit 2011 in Aachen bestehenden Veranstaltungsreihe mit begleitender Internetseite. In dieser Broschüre werden in einfühlsamer Sprache Themen, die Menschen mit einer Krebserkrankung während der Diagnosestellung, im Laufe der Therapie und in der Nachsorge beschäftigen, praxisnah und konkret behandelt. Die 5 inhaltlichen Kapitel beginnen alle mit dem Wort "Hoffnung" (Hoffnung auf ein gutes Leben während und nach einer Krebserkrankung, Hoffnung auf gesundes Leben, Hoffnung auf ein abgesichertes Leben, Hoffnung für spezielle Gruppen, Hoffnung auf Alltag), was den optimistischen und ganzheitlichen Duktus des Ratgebers verdeutlicht. In Unterkapiteln werden alle denkbaren Themen zu konkreten Alltagsfragen (z.B. Tipps zum ArztPatient Gespräch, Bewältigungsstrategien von Nebenwirkungen, Komplementärmedizin, Sozialrecht, Patientenverfügung etc.) konkret adressiert. Daneben werden weiter reichende Themen zu Fragen, die über die Krebsdiagnose hinausgehen, in tröstender und hilfreicher Weise behandelt (z.B. Angst und Angstbewältigung, Spiritualität, Junge Erwachsene mit Krebs, Angehörige, Kinder krebskranker Eltern). Schließlich erhalten potentiell angstbesetzte Themen wie Sexualität oder Palliativmedizin ausreichend Raum, um ausführlich, tiefgründig und sehr einfühlsam behandelt zu werden.
Dem Ratgeber merkt man deutlich an, dass er von erfahrenen ärztlich, psychologisch, seelsorgerlich und sozialrechtlich Tätigen geschrieben ist. Medizinische Tipps sind evidenzbasiert und aktuell aber mit dem oft nötigen Spielraum, um eine persönliche Bewältigung beispielsweise von Nebenwirkungen zu ermöglichen. Die Themen allgemeiner Art sind so breit gehalten, dass für zu finden ist. Insgesamt tragen alle Kapitel dazu bei, viel Sicherheit zu schaffen, auch zu den Fragen, die sich viele nicht zu stellen trauen. Komplettiert wird die Broschüre wo möglich mit Hinweisen auf Internetseiten und weiterführende Literatur, Selbsthilfegruppen etc. sowie mit einem schlüssigen und hilfreichen Glossar.

Mir war es ein Vergnügen, diese RatgeberBroschüre zu lesen. Während der Lektüre stellte sich wiederholt der Wunsch ein, die Broschüre neben den Patienten und Patientinnen ebenfalls allen Personen, die professionell in der Betreuung von Menschen mit Krebserkrankungen tätig sind, ans Herz zu legen und nicht zuletzt auch in den Unterricht von Studierenden zu integrieren.

M. von Lilienfeld-Toal, Jena (fast) jeden Geschmack etwas Interessantes 